\title{
Location based spectrum sensing evaluation in cognitive radio networks
}

\author{
Haipeng Yao ${ }^{*}$, Chenglin Zhao and Zheng Zhou*
}

\begin{abstract}
This letter addresses the problem of spectrum sensing over fading channel, in which a licensee and multiple unlicensed users coexist and operate in the licensed channel in a local area. We derive the overall average probabilities of detection and false alarm by jointly taking the fading and the location of SUs into account and employing the energy detection as the underlying detection scheme. Furthermore, we develop a statistical model of cumulate interference by the help of the overall average probabilities of detection. Based on the cumulate interference, we also obtain a closed-form expression of outage probability at the primary user's receiver according to a specific distribution of the fading.
\end{abstract}

Keywords: Spectrum sensing, cognitive radio, cumulate interference, outage probability

\section{Introduction}

The radio spectrum scarcity is becoming a serious problem as the consumers' increasing interest in wireless services. However, statistics show that most of the licensed frequency bands are severely underutilized across time and space in the sense that each licensee is granted an exclusive license to operate in a certain frequency band. The cognitive radio (CR), which was first proposed by Mitola [1], is a promising approach to solve the problem of imbalance between the spectrum scarcity and low utilization. The main idea contained in $\mathrm{CR}$ technology is that the secondary user (SU) can sense and exploit temporarily and available licensed spectrum and adapt its radio parameter to communicate over the spectrum of interest without harmfully interfering with the ongoing primary user (PU).

As the first step enabling the SUs sharing the spectrum with the PU, the spectrum sensing component needs to reliably and autonomously identify unused frequency bands. In general, spectrum sensing approaches can be classified into three categories; energy detection, matched filter coherent detection, and cyclostationary feature detection $[2,3]$. In this context, we choose the simple energy detection as the underlying detection

\footnotetext{
* Correspondence: yaohaipengbupt@gmail.com; zzhou@bupt.edu.cn Key Lab of Universal Wireless Communications, MOE, Wireless Network Lab, Beijing University of Posts and Telecommunications, Beijing, Peoples
} Republic of China scheme due to its low deployment cost and the ability of detecting any unknown signals.

One of the great challenges when we implement spectrum sensing is the uncertainty in probabilities of detection and false alarm which in turn results from the multipath fading or shadowing suffered by the SUs. Moreover, in the context of opportunistic spectrum access based on spectrum sensing, the uncertainty in the probability of false-alarm determines the percentage of the white spaces that are misclassified as occupied. Thus, a high probability of false-alarm in turn results in low spectrum utilization.

There are several previous works addressing the above issues. For example, in [4], a survey of spectrum sensing methodologies for cognitive radio was presented, and various aspects of spectrum sensing problem was studied from a cognitive radio perspective and multidimensional spectrum sensing concept was introduced. A statistical model of interference aggregation in spectrum-sensing cognitive radio networks was developed in [5]. However, the authors did not consider the optimization problem of the spectrum sensing parameters. The probabilities of detection and false alarm over fading channel were addressed in [6], and some alternative closed-form expressions for the probabilities of detection and false alarm were presented.

In this article, we will investigate the spectrum sensing performance from the perspective of the network level. 
In particular, for facilitating the design of the CR network, we derive the overall average probabilities of detection and false alarm jointly taking the fading and the location of SUs into account, i.e., the probabilities of detection and false alarm are averaged over all fading states and all locations of SUs. Then, we develop a statistical model of cumulate interference based on the above overall average probabilities of detection placed in a field of SUs, and derive the closed-form expression of outage probability at PU receiver based on a distribution of the fading.

The organization of this article is summarized as follows. We will put our contribution into context by giving a brief description of the system model and formulating the problems in 'System model' section. 'Interference modeling' section depicts the details of interference modeling. Our simulation results are given in 'Simulation results' section. Finally, we conclude this article in 'Concluding remarks' section.

\section{System model}

\section{Cognitive radio network model}

The cognitive radio network we considered here is shown in Figure 1. We model a situation where the SUs, each formed by a single transmitter-receiver pair, coexist and operate in a local circular region with a PU, and the radius is denoted by $R_{\mathrm{a}}$. The PU's receiver (PU $\mathrm{Rx}$ ) with omnidirectional antenna is assumed to be the center of the region. SUs satisfy uniform distribution in this region and the number of SUs is distributed according to a homogeneous Spatial Poisson process with density $\lambda$. Thus, the probability that there exist $k$ SUs in a region covering an area of $S$ is given by

$$
\operatorname{Pr}(k)=\frac{e^{-\lambda S}(\lambda S)^{K}}{k !} .
$$

Moreover, let $\mathrm{p}(r)$ denote the path-loss suffered by a signal of a transmitter at a distance $r$, and it can be expressed as

$$
\mathrm{p}(r)=\frac{1}{r^{\alpha}}
$$

where $\alpha>2$ is the path loss exponent. Note that this model is not feasible for the case $r<1$. In practical setting, however, the minimum physical distance $\left(R_{\min }\right)$ between the radios holds a natural constrains on $r$. Thus, we assume that $\mathrm{r} \geq R_{\min }$, without loss of generality, we only consider $R_{\min }=10$ in the remainder of this article. We further model the propagation power loss at a distance $r$ from the transmitter in fading channel as $p(r) X$, where $X \in \mathbb{R}^{+}$denotes the frequency-flat fading effect. Furthermore, we assume $X$ to be a unit-mean random variable and follow independent and identically distribution (i.i.d) for different SUs with $f_{\mathrm{x}}(x)$ and $F_{\mathrm{x}}(x)$ representing the probability density function (PDF) and the cumulative distribution function (CDF), respectively. $X$ is also assumed to be independent of the PU Rx's location.

\section{Spectrum sensing scheme}

We consider a spectrum sharing scheme in which the SUs are allowed to access the unused licensed spectrum without adversely interfering with the PU Rx. One of the central tasks in the spectrum sharing scheme is spectrum opportunity detection through sensing. Here, we assume the SU periodically detects the PU's transmitted signal in the licensed channel. By this method, the SUs can determine their behaviors, i.e., transmission over the licensed band or otherwise.

Here we employ the energy detection as the underlying detection scheme. An energy detector simply measures the energy received on the licensed channel during an observation interval and declares a white space if the measured energy is less than a proper threshold. Therefore, the spectrum sensing problem may be modeled as a binary hypothesis problem:

$H_{0}$ : The PU is absent,

$H_{1}$ : The PU is present.

Furthermore, we assume that the SUs carry out the spectrum sensing with energy detectors independently. The spectrum sensing with energy detection is to decide between the following two hypotheses,

$$
x_{i}(t)=\left\{\begin{array}{lc}
n_{i}(t), & H_{0} \\
h_{i} s_{\mathrm{p}}(t)+n_{i}(t), & H_{1}
\end{array}\right.
$$

where $x_{i}(t)$ is the received signal at $\mathrm{SU}_{i}, \mathrm{~S}_{\mathrm{p}}(t)$ is the PU's transmitted signal, $n_{i}(t)$ is the additive white Gaussian noise (AWGN), $h_{i}$ is the channel gain between the PU's transmitter and the $\mathrm{SU}_{i}$ 's receiver. Let $P_{\mathrm{p}}$ denotes the transmitted power of PU, the received instantaneous signal-to-noise ratio (SNR) at $\mathrm{SU}_{i}$ is defined as follows,

$$
\gamma_{i}=\frac{P_{\mathrm{p}} p\left(r_{i}\right) x_{i}}{N_{i}}
$$

where $x_{i}$ is the $\mathrm{SU}_{i}$ 's frequency-flat channel fading, $r_{i}$ denotes the distance between $\mathrm{SU}_{i}$ 's transmitter and the PU RX, $N_{i}$ is the power of AWGN. We denote by $\xi_{i}$ the collected energy which serve as decision statistic (where $\xi_{i}$ is defined as $\xi_{i}=\frac{1}{M} \sum_{j=1}^{M} x^{2}{ }_{i}(n), M$ is the number of sampling). Following by the work [7], the distribution of $\xi_{i}$ is

$$
\xi_{i} \sim\left\{\begin{array}{cc}
\chi_{2 m}^{2}, & H_{0} \\
\chi_{2 m}^{2}\left(2 \gamma_{i}\right) & , H_{1}
\end{array}\right.
$$




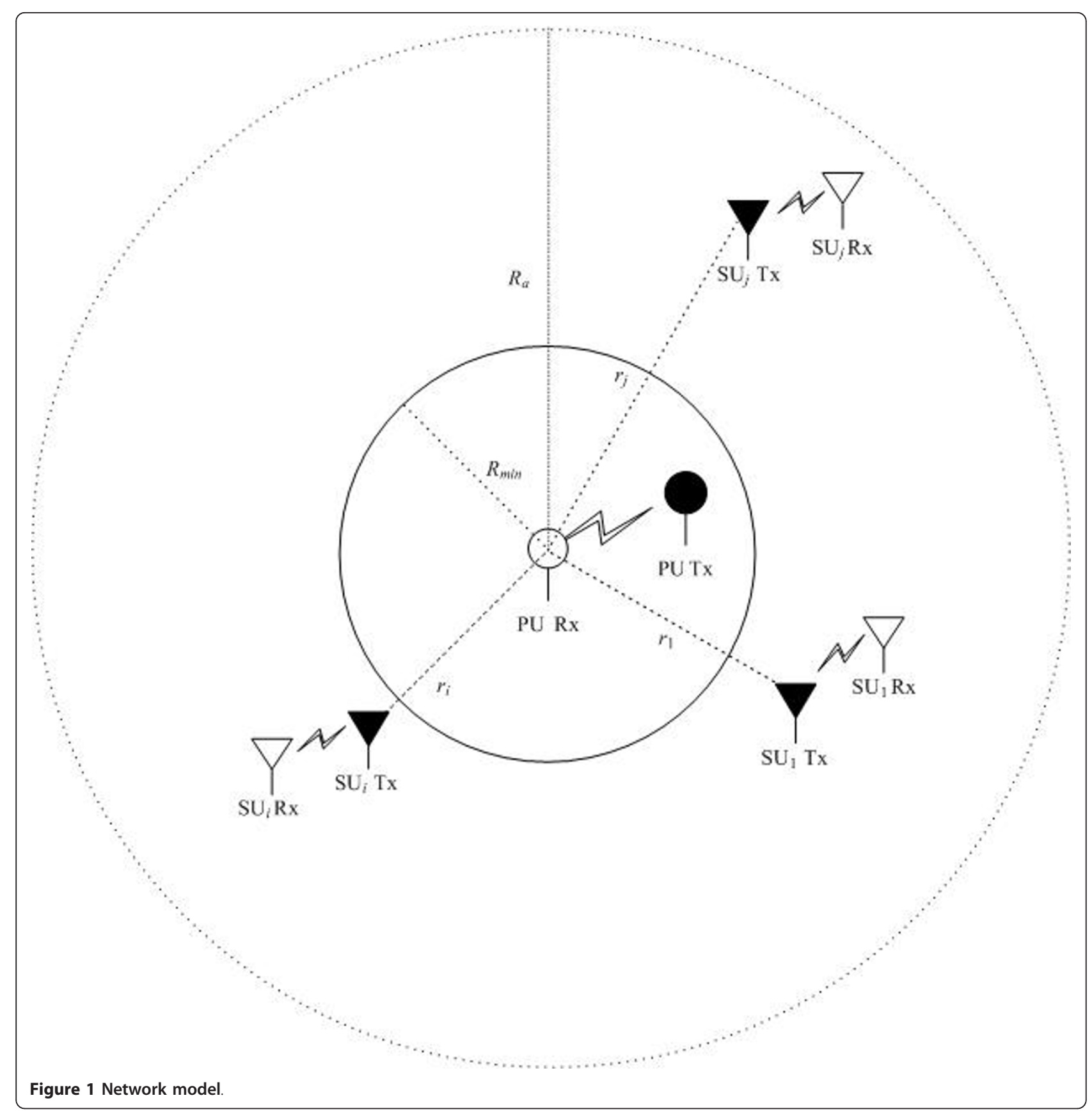

Where $\chi_{2 m}^{2}$ and $\chi_{2 m}^{2}\left(2 \gamma_{i}\right)$ denote the central and noncentral chi-square distribution, respectively, each with $2 m$ degrees of freedom and a non-centrality parameter $2 \gamma_{i}$ for $H_{1}$. Note that $m=T W$ is the time-bandwidth product, and for simplicity, it is assumed to be an integer.

The average probabilities of detection and false alarm for $\mathrm{SU}_{i}$ over a fading channel are given by the following equations, respectively,

$$
\begin{aligned}
P_{\mathrm{d}, i}=P\left(\xi_{i}>\tau_{i} \mid H_{1}\right) & =\int_{X} Q_{m}\left(\sqrt{2 \gamma_{i}}, \sqrt{\tau_{i}}\right) f_{\gamma_{i}}(x) \mathrm{d} x, \\
P_{\mathrm{f}, i}=P\left(\xi_{i}>\tau_{i} \mid H_{0}\right) & =\int_{x} \frac{\Gamma\left(m, \tau_{i} / 2\right)}{\Gamma(m)} f_{\gamma_{i}}(x) \mathrm{d} x \\
& =\frac{\Gamma\left(m, \tau_{i} / 2\right)}{\Gamma(m)}
\end{aligned}
$$


where $\tau_{i}$ denotes $\mathrm{SU}_{i}$ 's energy detection threshold, $\Gamma($.) and $\Gamma(.,$.$) are complete and upper incomplete gamma$ function, respectively, $f_{\gamma i}(x)$ is the PDF of $\gamma_{i}$ under fading, $x$ denotes the frequency-flat channel fading, $Q_{m}(\mu$, $v$ ) denotes the generalized Marcum Q-function defined as follows,

$$
Q_{m}(\mu, v)=\frac{1}{\mu^{m-1}} \int_{v}^{\infty} x^{m} \exp \left(-\frac{x^{2}+\mu^{2}}{2}\right) I_{m-1}(\mu x)(B x),
$$

where $I_{m-1}($.$) is the modified Bessel function of the first$ kind and order $m-1$. We note that (7) is derived due to the fact that $\Gamma\left(m, \tau_{i} / 2\right) / \Gamma(m)$ is independent of $\gamma_{i}$.

Moreover, since the number of SUs follows homogeneous Spatial Poisson process, the probability that the $\mathrm{SU}$ is at a distance $r$ from the PU Rx may expressed as

$$
f(r)=\frac{2 r}{D} \quad R_{\min } \leq r \leq R_{\mathrm{a}}
$$

with $D=R_{\mathrm{a}}^{2}-R_{\mathrm{min}}^{2}$. Let $P_{d}$ and $P_{f}$, be $P_{d, i}$ and $P_{\mathrm{f}, i}$ averaged over all locations of SUs, respectively, and we assume that all SUs use the different decision rule, for simplifying the following discussing, we assume that the mean of the SUs' energy threshold is $\tau$, i.e., $\tau=E\left(\tau_{i}\right)$. Then, $P_{d}$ and $P_{\mathrm{f}}$ can be calculated by

$$
\begin{aligned}
& P_{\mathrm{d}}=E\left(P_{\mathrm{d}, i}\right), \\
& P_{\mathrm{f}}=E\left(P_{\mathrm{f}, i}\right)=E\left(\frac{\Gamma(m, \tau / 2)}{\Gamma(m)}\right)=\frac{\Gamma(m, \tau / 2)}{\Gamma(m)},
\end{aligned}
$$

where $E($.$) denotes the expectation. Furthermore, (10)$ can be calculated by conditioning on the number of SUs, i.e.,

$$
E\left(P_{\mathrm{d}, i}\right)=\sum_{k=0}^{\infty} \frac{\mathrm{e}^{-\lambda \pi D}(\lambda \pi D)^{k}}{k !} E\left(P_{\mathrm{d}, i} \mid k \text { SUs }\right) .
$$

By plugging (9) into (12), after some manipulation, we have

$$
\begin{aligned}
E\left(P_{\mathrm{d}, i}\right) & =\sum_{k=0}^{\infty} \frac{\mathrm{e}^{-\lambda \pi D}(\lambda \pi D)^{k}}{k !} E\left(P_{\mathrm{d}, i} \mid k \text { SUs }\right) \\
& =\sum_{k=0}^{\infty} \frac{\mathrm{e}^{-\lambda \pi D}(\lambda \pi D)^{k}}{k !} E_{1}\left(P_{\mathrm{d}}\right)^{k} \\
& =\mathrm{e}^{\lambda \pi D\left(E_{1}\left(P_{\mathrm{d}}\right)-1\right),}
\end{aligned}
$$

where the third line of (13) is obtained due to the fact that $\sum_{l=0}^{\infty} \frac{\mathrm{e}^{-\sigma}}{l !}(\sigma)^{l}=1$, and $E_{1}\left(P_{\mathrm{d}}\right)$ may be calculated by

$$
E_{1}\left(P_{\mathrm{d}}\right)=\int_{X} f_{\gamma_{i}}(x) \mathrm{d} x \int_{R_{\min }}^{R_{\mathrm{a}}} Q_{m}\left(\sqrt{2 \gamma_{i}}, \sqrt{\tau}\right) \frac{2 r}{D} \mathrm{~d} r
$$

We can investigate that both $p_{\mathrm{d}}$ and $p_{\mathrm{f}}$ are functions in term of $\tau$, and can be denoted by $P_{\mathrm{d}}(\tau)$ and $P_{\mathrm{f}}(\tau)$, respectively.

\section{Interference modeling}

To enable the spectrum sharing with PU, many problems remain to be solved. Most importantly, the SUs have to make sure they do not cause unacceptable interference to PU. In this section, we will develop a statistical model of interference aggregation caused by the SUs.

The interference suffering by the PU is mainly caused by the SU's behavior of missed detection of the PU's signals. For facilitating the following discussion, the overall average probability of missed detection may be written as $P_{\mathrm{m}}(\tau)=1-P_{\mathrm{d}}(\tau)$.

According to the earlier description about the distribution of SUs, let $\Pi_{I}$ denotes the set of interfering SUs, it can be easy proved that $\Pi_{I}$ forms a homogeneous Spatial Poisson process with density $\lambda P_{m}(\tau)$. Thus, the cumulate interference caused by SUs in $\Pi_{I}$ can be expressed as

$$
I_{T}=\sum_{i \in \Pi_{I}} P_{\mathrm{Si}} p\left(r_{i}\right) x_{i}
$$

Where $P_{S i}$ represents the $\mathrm{SU}_{i}^{\prime}$ s transmitted power.

In the subsection, we follow the routine in [8] to obtain the CDF of (15). We will first derive the characteristic function of $I_{T}$. By the definition, the characteristic function of $I_{T}$ is given by

$$
\psi_{I_{T}}(w)=E\left(\mathrm{e}^{j w I_{T}}\right) .
$$

Once again using the similar method described in 'System model' section, (16) can be calculated by the following equation,

$$
E\left(\mathrm{e}^{j w I_{T}}\right)=E\left(E\left(\mathrm{e}^{\mathrm{j} w I_{T}} \mid l \text { in } \Pi_{I}\right)\right) .
$$

Considering the fact that SUs in $\Pi_{I}$ following homogeneous Spatial Poisson process with density $\lambda P_{\mathrm{m}}(\tau), \mathrm{E}$ $\left(\mathrm{e}^{j w I T}\right)$ can be further calculated by

$$
E\left(\mathrm{e}^{j w I_{T}}\right)=\sum_{l=0}^{\infty} \frac{\mathrm{e}^{-\lambda P_{\mathrm{m}}(\tau) \pi D}\left(\lambda P_{\mathrm{m}}(\tau) \pi D\right)^{l}}{l !} E\left(\mathrm{e}^{\mathrm{j} w I_{T}} \mid l \text { in (III } /\right) .
$$

In what follows, for easy of exposition, we assume that the SUs adopt the different transmitted power, and the mean of the SUs' energy threshold is $P_{c}$, i.e., $P_{C}=E\left(P_{S i}\right)$. In what follows, we adopt $P_{C}$ to value the performance. Thus, (18) can be rewritten as 


$$
\begin{gathered}
\sum_{l=0}^{\infty} \frac{\mathrm{e}^{-\lambda P_{\mathrm{m}}(\tau) \pi D}\left(\lambda P_{\mathrm{m}}(\tau) \pi D\right)^{l}}{l !} E\left(\mathrm{e}^{j \omega I_{T}} \mid l \text { in } \Pi_{I}\right) \\
=\sum_{l=0}^{\infty} \frac{\mathrm{e}^{-\lambda P_{\mathrm{m}}(\tau) \pi D}\left(\lambda P_{\mathrm{m}}(\tau) \pi D\right)^{l}}{l !}\left[E_{2}\left(\mathrm{e}^{j w P_{\mathrm{C} P}(r) X}\right)\right]^{l} \\
=\mathrm{e}^{\lambda P_{\mathrm{m}}(\tau) \pi D\left(E_{2}\left(e^{\left.j \omega P_{\mathrm{C}}(r) X\right)}\right)-1\right),}
\end{gathered}
$$

where $E_{2}($.$) denotes the expectation about I_{T}$, and can be calculated by

$$
E_{2}\left(\mathrm{e}^{j w P_{\mathrm{C}} p(r) X}\right)=\int_{X} f_{\mathrm{X}}(x) \int_{R_{\min }}^{R_{\mathrm{a}}} \mathrm{e}^{j w P_{\mathrm{C}} p(r) x} \frac{2 r}{D} \mathrm{~d} r \mathrm{~d} x .
$$

Since (20) is not easy to be simplified, we generally cannot derive the exact closed-form expression of the characteristic function as well as the distribution of the cumulate interference. However, we can approximate the distribution of the cumulate interference by deriving the cumulants of the interference. The $k$ th cumulant, $\eta_{k}$, is given by

$$
\begin{aligned}
\eta_{k} & =\left.\left[\frac{1}{j^{k}} \frac{\partial^{k} \ln \psi_{I_{T}}(w)}{\partial w^{k}}\right]\right|_{w=0} \\
& =2 \lambda \pi P_{\mathrm{C}}^{k} P_{\mathrm{m}}(\tau) \int_{X} f_{\mathrm{X}}(x) \int_{R_{\min }}^{R_{\mathrm{a}}} x^{k} r^{1-k \alpha} \mathrm{d} r \mathrm{~d} x \\
& =2 \lambda \pi P_{\mathrm{C}}^{k} P_{\mathrm{m}}(\tau) \int_{X} x^{k} f_{\mathrm{X}}(x) \int_{R_{\min }}^{R_{\mathrm{a}}} r^{1-k \alpha} \mathrm{d} r \mathrm{~d} x \\
& =\frac{2 \lambda \pi P_{\mathrm{C}}^{k} P_{\mathrm{m}}(\tau)}{2-k \alpha} E\left(X^{k}\right)\left(R_{\mathrm{a}}^{2-k \alpha}-R_{\min }^{2-k \alpha}\right)
\end{aligned}
$$

\section{Simulation results}

In this section, we present the application of the formulas constructed in the previous sections through some additional numerical simulation. More specifically, we

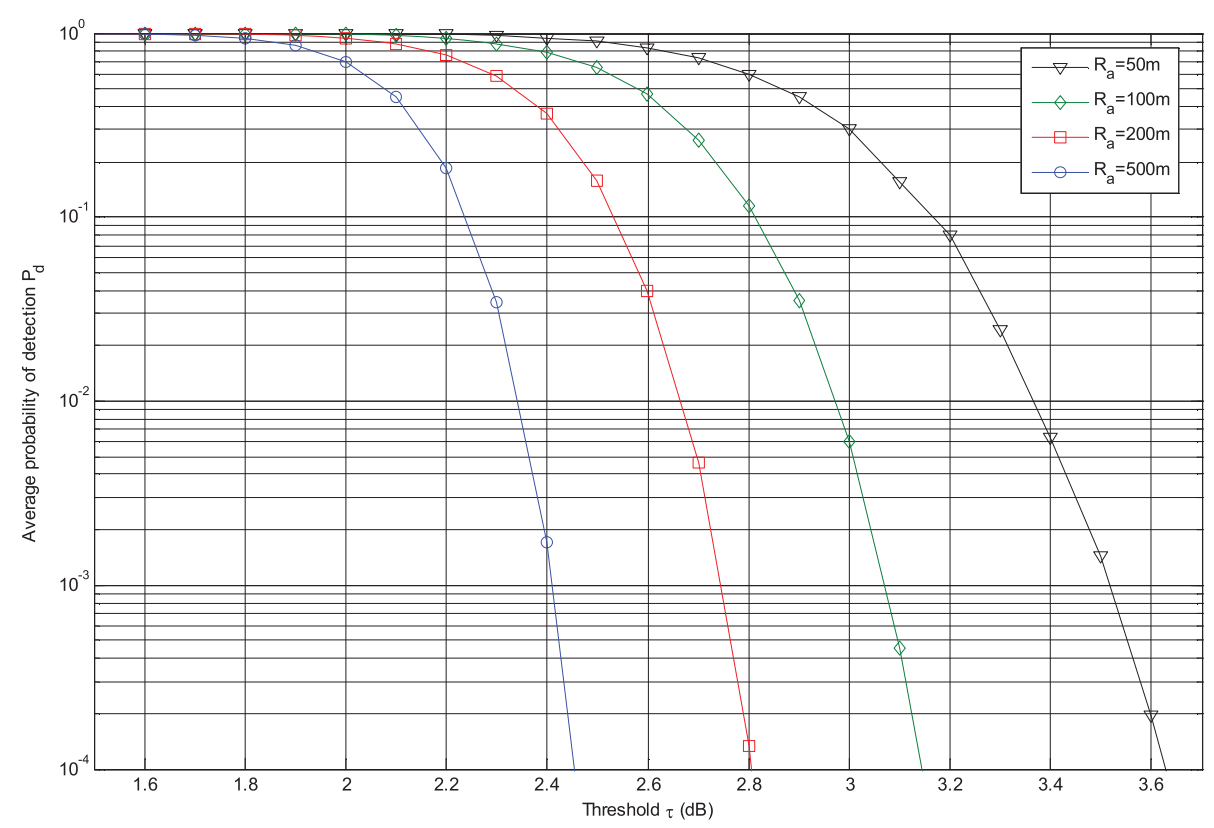

Figure $2 P_{\mathrm{d}}$ vs. $\tau$ under log-normal shadowing for different radii of the network ( $\sigma_{\mathrm{dB}}=6 \mathrm{~dB}, \alpha=4, \lambda=0.01, m=10$ ). 
are interested in investigating the relationship between the overall average probability of detection and the threshold. We also study the impact of the CR network scale on the probability of detection and the outage at the PU Rx.

Figure 2 shows the overall average probability of detection as a function of the detection threshold for different radii of the network. $P_{\mathrm{p}}$ is assumed to be 10 $\mathrm{dB}$. As expected, increasing the detection threshold would significantly reduce the average probability of detection. We also observe that increasing the radius of the network deteriorates the average detection performance. In fact, for a lager scale network the PU's signal is difficult to be detected for those kinds SUs located far from the PU Rx.

Figure 3 depicts the outage probability at PU Rx in terms of the detection threshold for different radii of the network. As before $P_{\mathrm{c}}$ is assumed to be $10 \mathrm{~dB}$. As seen in Figure 3, with increasing $\tau$, the outage probability tends to be worse. Moreover, the outage probability with a small radius of the network (i.e., $R_{a}=100$ ) is capable of outperforming that with a large radius of the network ( $i$. e., $\left.R_{a}=500\right)$. Consequently, the spectrum sensing with jointly taking the fading and location of SUs into account is more suitable for the small scale network.

\section{Concluding remarks}

Spectrum sensing is viewed as a crucial component of the emerging cognitive radio networks. In this article, we study the spectrum sensing problem jointly taking the fading and the location of SUs into account. We obtain the overall average probabilities of detection and false alarm, and further construct the model of cumulate interference.

\section{List of Abbreviations}

AWGN: additive white Gaussian noise; CDF: cumulative distribution function; CR: cognitive radio; i.i.d: independent and identically distribution; PDF: probability density function; PU: primary user; PU Rx: PU's receiver; SNR: signal-to-noise ratio; SU: secondary user.

\section{Acknowledgements}

This research was partly supported by the Ministry of Knowledge Economy, Korea, under the ITRC support program supervised by the Institute for Information Technology Advancement (IITA-2009-C1090-0902-0019). This work was supported by following projects: NSFC (60772021), The Research Fund for the Doctoral Program of Higher Education (20060013008, 20070013029), and the National High-tech Research and Development Program (863 Program) (2009AA01Z262).

\section{Competing interests}

The authors declare that they have no competing interests.

Received: 14 March 2011 Accepted: 24 August 2011

Published: 24 August 2011

\section{References}

1. J Mitola III, G Maguire Jr, Cognitive radio: making software radios more personal. Personal Communications, IEEE see also IEEE Wire Commun. 6(4), 13-18 (1999)

2. H Urkowitz, Energy detection of unknown deterministic signals. Proc IEEE. 55, 523-231 (1967)

3. D Cabric, SM Mishra, RW Brodersen, Implementation issues in spectrum sensing for cognitive radios, in Proceedings of Asilomar Conference Signals, Systems, and Computers, 772-76 (Nov. 2004)

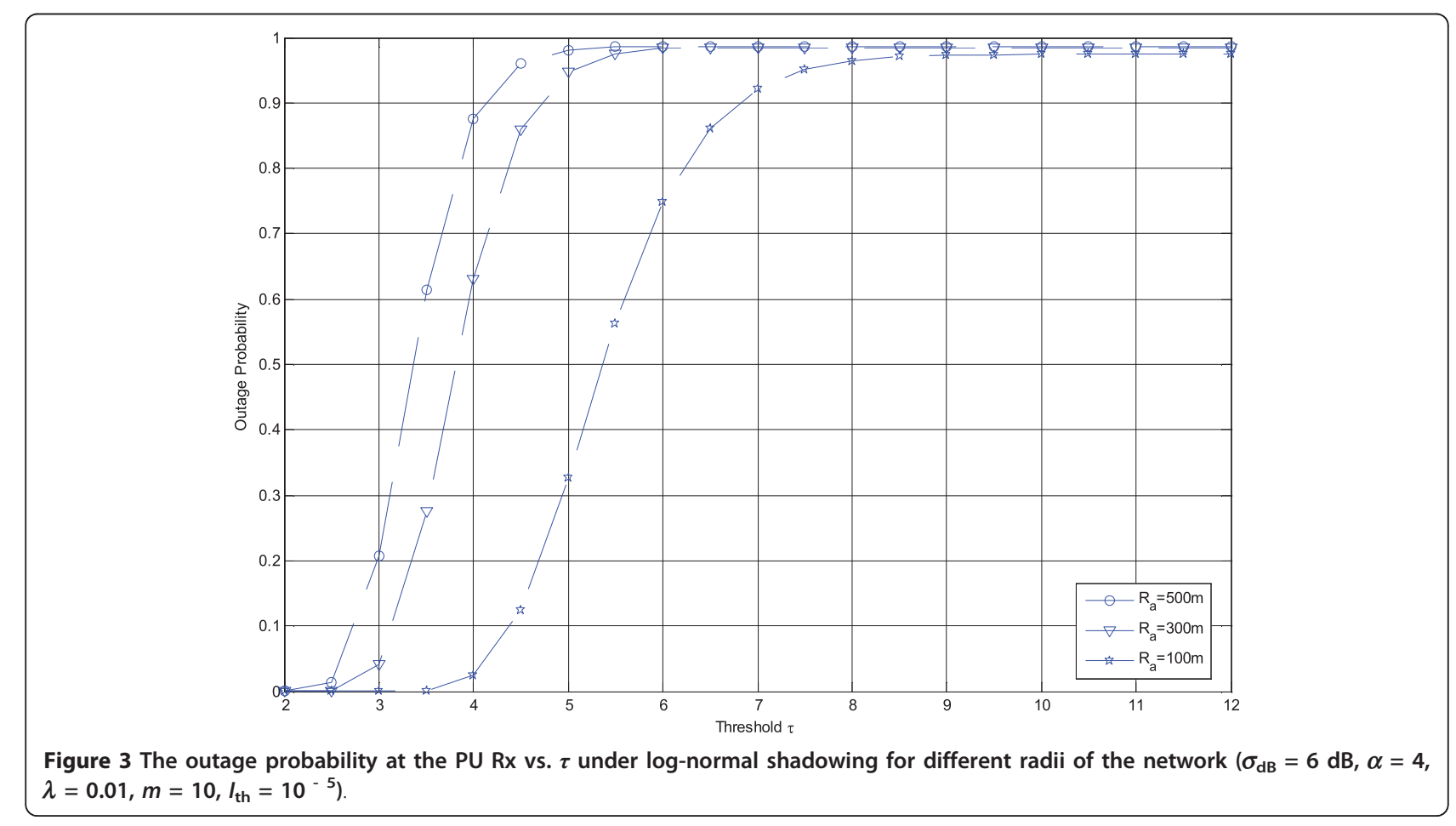


4. T Yucek, $H$ Arslan, A survey of spectrum sensing algorithms for cognitive radio applications. IEEE Commun Surveys Tutorials. 11(1), 772-776 (2009)

5. A Ghasemi, ES Sousa, Interference aggregation in spectrum-sensing cognitive wireless networks. IEEE J Select Topics Signal Process. 2(1), 41-55 (2008)

6. FF Digham, MS Alouini, MK Simon, On the energy detection of unknown signals over fading channels. IEEE Trans Commun. 55(1), 3575-3579 (2007)

7. VI Kostylev, Energy detection of a signal with random amplitude, in Proceedings of IEEE International Conference on Communication, (New York), pp. 1606-1610 (28 Apr-may 2002)

8. ES Sousa, JA Silvester, Optimum transmission ranges in adirect-sequence spread-spectrum multihop packet radio network. IEEE J Select Areas Commun. 8(5), 762-771 (1990). doi:10.1109/49.56383

9. TS Rappaport, Wireless Communication Principles and Practice, 2nd edn. (Prentice Hall, Upper Saddle River, NJ, 2002)

\section{doi:10.1186/1687-1499-2011-74}

Cite this article as: Yao et al:: Location based spectrum sensing evaluation in cognitive radio networks. EURASIP Journal on Wireless Communications and Networking $20112011: 74$.

\section{Submit your manuscript to a SpringerOpen ${ }^{\circ}$ journal and benefit from:}

- Convenient online submission

- Rigorous peer review

- Immediate publication on acceptance

- Open access: articles freely available online

- High visibility within the field

- Retaining the copyright to your article

Submit your next manuscript at $\gg$ springeropen.com 